Скопје, Македонија

\title{
ABOUT THE ACCORDANCE BETWEEN THE CANONICAL VEKUA DIFFERENTIAL EQUATION AND THE GENERALIZED HOMOGENEOUS DIFFERENTIAL EQUATION
}

\author{
Slagjana Brsakoska
}

\begin{abstract}
In the paper two equations, the canonical Vekua differential equation and the generalized homogeneous differential equation, are considered. The main result is the theorem with the condition for the accordance between this two equations.
\end{abstract}

\section{INTRODUCTION}

The equation

$$
\frac{\hat{d} W}{d \bar{z}}=A W+B \bar{W}+F
$$

where $A=A(z), B=B(z)$ and $F=F(z)$ are given complex functions from a complex variable $z \in D \subseteq \mathbb{C}$ is the well known Vekua equation [1] according to the unknown function $W=W(z)=u+i v$. The derivative on the left side of this equation has been introduced by G.V. Kolosov in 1909 [2]. During his work on a problem from the theory of elasticity, he introduced the expressions

$$
\frac{1}{2}\left[\frac{\partial u}{\partial x}+\frac{\partial v}{\partial y}+i\left(\frac{\partial v}{\partial x}-\frac{\partial u}{\partial y}\right)\right]=\frac{\hat{d} W}{d z}
$$

and

$$
\frac{1}{2}\left[\frac{\partial u}{\partial x}-\frac{\partial v}{\partial y}+i\left(\frac{\partial v}{\partial x}+\frac{\partial u}{\partial y}\right)\right]=\frac{\hat{d} W}{d \bar{z}}
$$

2010 Mathematics Subject Classification. 34M45, 35Q74.

Key words and phrases. areolar derivative, areolar equation, analytic function, Vekua equation, generalized homogeneous differential equation. 
known as operator derivatives of a complex function $W=W(z)=u(x, y)+$ $i v(x, y)$ from a complex variable $z=x+i y$ and $\bar{z}=x-i y$ corresponding. The operating rules for this derivatives are completely given in the monograph of $\Gamma$. Н.Положий [3] (page18-31). In the mentioned monograph are defined so cold operator integrals $\hat{\int} f(z) d z$ and $\hat{\int} f(z) d \bar{z}$ from $z=x+i y$ and $\bar{z}=x-i y$ corresponding (page 32-41). As for the complex integration in the same monograph is emphasized that it is assumed that all operator integrals can be solved in the area D.

In the Vekua equation (1) the unknown function $W=W(z)$ is under the sign of a complex conjugation which is equivalent to the fact that $B=B(z)$ is not identically equaled to zero in D. That is why for (1) the quadratures that we have for the equations where the unknown function $W=W(z)$ is not under the sign of a complex conjugation, stop existing.

This equation is important not only for the fact that it came from a practical problem, but also because depending on the coefficients $\mathrm{A}, \mathrm{B}$ and $\mathrm{F}$ the equation (1) defines different classes of generalized analytic functions. For example, for $F=F(z) \equiv 0$ in $\mathrm{D}$ the equation (1) i.e.

$$
\frac{\hat{d} W}{d \bar{z}}=A W+B \bar{W}
$$

which is called canonical Vekua equation, defines so cold generalized analytic functions from fourth class; and for $A \equiv 0$ and $F \equiv 0$ in D, the equation (1) i.e. the equation $\frac{\hat{d} W}{d \bar{z}}=B \bar{W}$ defines so cold generalized analytic functions from third class or the ( $\mathrm{r}+\mathrm{is})$-analytic functions [3], [4].

Those are the cases when $B \neq 0$. But if we put $B \equiv 0$, we get the following special cases. In the case $A \equiv 0, B \equiv 0$ and $F \equiv 0$ in the working area $D \subseteq \mathbb{C}$ the equation (1) takes the following expression $\frac{\hat{d} W}{d \bar{z}}=0$ and this equation, in the class of the functions $W=u(x, y)+i v(x, y)$ whose real and imaginary parts have unbroken partial derivatives $u_{x}^{\prime}, u_{y}^{\prime}, v_{x}^{\prime}$ and $v_{y}^{\prime}$ in $\mathrm{D}$, is a complex writing of the Cauchy - Riemann conditions. In other words it defines the analytic functions in the sense of the classic theory of the analytic functions. In the case $B \equiv 0$ in D i.e. $\frac{\hat{d} W}{d \bar{z}}=A W+F$ is the so cold areolar linear differential equation [3] (page 39-40) and it can be solved with quadratures by the formula: 


$$
W=e^{\hat{\int} A(z) d \bar{z}}\left[\Phi(z)+\hat{\int} F(z) e^{-\hat{\int} A(z) d \bar{z}} d \bar{z}\right]
$$

Here $\Phi=\Phi(z)$ is an arbitrary analytic function in the role of an integral constant.

\section{FORMULATION OF THE PROBLEM AND MAIN RESULT}

In the paper [5], the following lemma is proved.

Lemma. The equations

$$
\frac{\hat{d} W}{d z}=f(z, W)
$$

and

$$
\frac{\hat{d} W}{d \bar{z}}=g(z, W)
$$

where $\frac{\hat{d} f}{d \bar{W}}=0$, have common solutions if and only if

$$
\frac{\hat{d} f}{d \bar{z}}+\frac{\hat{d} f}{d W} g=\frac{\hat{d} g}{d z}+\frac{\hat{d} g}{d W} f+\frac{\hat{d} g}{d \bar{W}} \bar{g} .
$$

It is assumed that the operator derivatives in (7) exist and that they are continuous functions in the working area $\mathrm{D}$ from the complex plane.

In this paper we are examining the accordance between the canonical Vekua equation (4), on one side and the generalized homogeneous differential equation

$$
\frac{\hat{d} W}{d z}=\varphi\left(\frac{W}{z}\right)
$$

on the other side, where $\varphi=\varphi(z)$ is a given complex functions from a complex variable $z \in D \subseteq \mathbb{C}$, such that $\frac{\hat{d} \varphi}{d \bar{W}}=0$. The canonical Vekua equation (4) is an equation of type (6) where

$$
g(z, W)=A W+B \bar{W}
$$

and the generalized homogeneous differential equation (8) is an equation of type (5), where

$$
f(z, W)=\varphi\left(\frac{W}{z}\right)
$$


Here, the function $f$ is an analytic function according to $W$, which means that $\frac{\hat{d} f}{d \bar{W}}=0$. That is the only condition to be accomplished, so that we can use the mentioned lemma.

If we calculate all the derivatives in (7), we get that

$$
\begin{array}{lll}
\frac{\hat{d} f}{d \bar{z}}=\frac{\hat{d} \varphi}{d \bar{z}}, & \frac{\hat{d} f}{d W}=\frac{\hat{d}}{d W} \varphi\left(\frac{W}{z}\right)=\frac{\hat{d} \varphi}{d W} \cdot \frac{1}{z}, \\
\frac{\hat{d} g}{d z}=\frac{\hat{d} A}{d z} W+\frac{\hat{d} B}{d z} \bar{W}, & \frac{\hat{d} g}{d W}=A, & \frac{\hat{d} g}{d \bar{W}}=B .
\end{array}
$$

And if we put them in (7) we get that

$$
\left.\frac{\hat{d} \varphi}{d \bar{z}}+\frac{\hat{d} \varphi}{d W} \cdot \frac{1}{z}(A W+B \bar{W})=\frac{\hat{d} A}{d z} W+\frac{\hat{d} B}{d z} \bar{W}+A \varphi+B \overline{(A W+B \bar{W}}\right) .
$$

Now we write the last equation in the following form

$$
W\left(\frac{\hat{d} \varphi}{d W} \cdot \frac{A}{z}-\frac{\hat{d} A}{d z}-|B|^{2}\right)+\bar{W}\left(\frac{\hat{d} \varphi}{d W} \cdot \frac{B}{z}-\frac{\hat{d} B}{d z}-\bar{A} B\right)+\frac{\hat{d} \varphi}{d \bar{z}}-A \varphi=0 .
$$

This linear combination is true only if the following system of equation is satisfied

$$
\left\{\begin{array}{l}
\frac{\hat{d} \varphi}{d W} \cdot \frac{A}{z}-\frac{\hat{d} A}{d z}-|B|^{2}=0 \\
\frac{\hat{d} \varphi}{d W} \cdot \frac{B}{z}-\frac{\hat{d} B}{d z}-\bar{A} B=0 . \\
\frac{\hat{d} \varphi}{d \bar{z}}-A \varphi=0
\end{array} .\right.
$$

If we eliminate the derivative $\frac{\hat{d} \varphi}{d W}$ from the first and the second equation in the system (11), we get

$$
\begin{aligned}
& \frac{\hat{d} \varphi}{d W}=\frac{z}{A}\left(\frac{\hat{d} A}{d z}+|B|^{2}\right) \\
& \frac{z}{A}\left(\frac{\hat{d} A}{d z}+|B|^{2}\right) \cdot \frac{B}{z}-\frac{\hat{d} B}{d z}-\bar{A} B=0 \\
& B \frac{\hat{d} A}{d z}-A \frac{\hat{d} B}{d z}+B|B|^{2}-B|A|^{2}=0
\end{aligned}
$$

Because of the fact that $\frac{\hat{d}}{d z}\left(\frac{A}{B}\right)=\frac{1}{B^{2}}\left(\frac{\hat{d} A}{d z} B-A \frac{\hat{d} B}{d z}\right)$, we get that

$$
\begin{aligned}
& B^{2} \frac{\hat{d}}{d z}\left(\frac{A}{B}\right)+B\left(|B|^{2}-|A|^{2}\right)=0 \\
& \frac{\hat{d}}{d z}\left(\frac{A}{B}\right)+\frac{|B|^{2}-|A|^{2}}{B}=0
\end{aligned}
$$

or

$$
\frac{\hat{d}}{d z}\left(\frac{A}{B}\right)+\bar{B}-\frac{A}{B} \bar{A}=0
$$


which is the condition between the coefficients in the Vekua equation (4) in order to has common solutions with the equation (8).

The third equation in the system (11) is an areolar equation which can be solved, i.e.

$$
\begin{aligned}
\frac{1}{\varphi} \cdot \frac{\hat{d} \varphi}{d \bar{z}} & =A \\
\ln \varphi \frac{W}{z} & =\hat{\int} A(z) d \bar{z}+\ln \Phi(z) \\
\varphi\left(\frac{W}{z}\right) & =\Phi(z) \cdot \exp \left(\hat{\int} A(z) d \bar{z}\right)
\end{aligned}
$$

Here $\Phi=\Phi(z)$ is an arbitrary analytic function in the role of an integral constant.

So, we have proved the following

Theorem. The Vekua equation (4) and the generalized homogeneous equation (8) have common solutions if and only if the condition (12) is fulfilled and the relation between the coefficients of the two equations are given with (13).

Note 1. The condition (12) that we got, works for example if $A=B$.

Note 2. In [6], we can see the condition between the coefficients in the Vekua equation (1) (and (4) also), in order to has common solutions with the generalized linear equation and the relation between its coefficients. If we compare the theorems, they have similar statement, but different conditions and relations that we mentioned. Further more, in [6] both the equations (1) and (4) are considered and in this case only the equation (4) is considered. This refers to the easier manipulation with the generalized linear equation in comparison with the generalized homogeneous equation. 


\section{References}

[1] Н. И. Векуа, Обобщение аналитические функциии, Москва,1988

[2] Г. В. Колосов, Об одном приложении теории функции комплесного переменного к плоское задаче математическои упругости, 1909

[3] Г. Н. Положии, Обопштение теории аналитических фукции комплесного переменного, Издателство Киевского Университета, 1965

[4] S. Brsakoska, Operator differential equations from the aspect of the generalized analytic functions, MSc thesis, Skopje, 2006

[5] B. Ilievski, S. Brsakoska, Za soglasnost na homogena ravenka Vekua $i$ obopštena diferencijalna ravenka od prv red (About an accordance of a homogenous Vekua equation and generalized differential equation of first order), III Congress of Mathematicians of Macedonia, Struga, 2005

[6] S. Brsakoska, About the accordance between the Vekua differential equation and the generalized linear differential equation, Zbornik radova Međunarodne konferencije Matematičke i informacione tehnologije, MIT 2013, Vrnjačka Banja, Srbija, 2013, str. 95-98.

Ss. Cyril and Methodius University

Faculty of Natural Sciences and Mathematics, Arhimedova 3, 1000 Skopje, R. Macedonia e-mail: sbrsakoska@gmail.com 